\title{
Effect of Main-Chain Rigidity on Fatigue Behavior of Polymeric Fibers Based on Zone Nonlinear Viscoelastic Analysis
}

\author{
Tao Liang, Atsushi Takahara, Koichi Saito, ${ }^{*}$ and Tisato KajIYama \\ Department of Chemical Science and Technology, Faculty of Engineering, Kyushu University, \\ Hakozaki, Higashi-ku, Fukuoka 812-81, Japan \\ *Central Research Laboratories, Kuraray Co., Ltd., Sakazu, Kurashiki, Okayama 710, Japan
}

(Received March 28, 1996)

\begin{abstract}
Fatigue behavior of high-strength and high-modulus polymeric fibers was investigated based on nonlinear dynamic viscoelastic analysis under tension-tension cyclic strain condition. Poly(vinyl alcohol) (PVA) and thermotropic polyester(Vectran) fibers were used as specimens. The zone nonlinear dynamic viscoelastic analysis was proposed as a new approach to investigate the nonlinear dynamic viscoelastic behavior in different deformation stages during one period of cyclic deformation for polymeric materials. It was found that the nonlinear dynamic viscoelasticity strongly depended on the rigidity of backbone chains of polymers. The polymeric fibers with rigid polymer chains showed remarkable nonlinear viscoelastic characteristics comparing with that with flexible polymer chains. Also, it was found from the zone nonlinear dynamic viscoelastic analyses that the nonlinear viscoelastic behavior during cyclic deformation was predominantly induced during the recovery process rather than the tensile process for polymeric fibers, and the more remarkable nonlinear viscoelastic behavior was exhibited in the zone with higher strain rate. The polymeric fibers with rigid polymer chains exhibited more remarkable nonlinear viscoelasticity and poorer fatigue strength. At the onset of fatigue failure, a sudden increase in nonlinear dynamic viscoelasticity was observed for polymeric fibers. The sudden increase was considered to relate with the remarkable irreversible structural changes before the fatigue failure for polymeric materials.

KEY WORDS Nonlinear Dynamic Viscoelasticity / Zone Nonlinear Dynamic Viscoelasticity / Fatigue / Rigidity of Polymer Chains / Main-Chain Liquid Crystalline Polymer /
\end{abstract}

During the past decades, as polymeric materials have been used in many load bearing structural applications wherein the reliability and safety are very important, an increasing amount of attention has been paid to the fatigue behavior of polymers. The fatigue behavior of polymers has thus far been investigated over a wide scale range using several different approaches. In most conditions, the specimens of polymers are simply subjected to various magnitudes of cyclic strain or stress, and the number of cycles up to fatigue failure is determined. ${ }^{1}$ Some researchers also used the specimens that had been precracked, and then, the crack growth rate was determined as a function of applied stress intensity factors. ${ }^{1}$ Such information, as baseline data, is very important and can provide engineers a crude guideline to design structure components. However, on molecular level, little effort has been made to clarify the nature and the origin of fatigue failure about polymeric materials.

As one of the most important mechanical properties of polymers, the viscoelasticity has been found to relate with the fatigue properties. ${ }^{2-5}$ Also, since in actual fatigue test, the imposed strain or stress must be large enough to ultimately lead to a fatigue failure, the nonlinear viscoelastic behavior cannot be ignored under such fatigue test condition. So it seems very important to investigate the fatigue behavior of polymeric materials based on the nonlinear dynamic viscoelastic analysis. ${ }^{3-5}$

Owing to no successful theoretical interpretation about the phenomena of nonlinear dynamic viscoelastic behavior, until now, the most accurate, reliable and also, reproducible approach for the nonlinear dynamic viscoelastic analysis has to be based on the direct observation of the nonlinear responses. ${ }^{6,7}$ In recent years, the direct analysis of the nonlinear dynamic viscoelastic responses with Fourier transformation ${ }^{3-5,8-9}$ has been paid an increasing attention because it does not need any extra theoretical hypothesis and can provide much reliable and reproducible conclusions. The authors have proposed an approach (which can be called the nonlinear viscoelastic parameter (NVP) analysis) for the quantitative evaluation on the nonlinear dynamic viscoelasticity. The nonlinear viscoelastic parameter, NVP, which enabled the evaluation of the degree of nonlinear dynamic viscoelasticity, could be simply calculated by the coefficients of the Fourier series transformed from the stress response. ${ }^{3-5}$

However, NVP provides only the information about the average or whole characteristics of nonlinear dynamic viscoelasticity during one cyclic deformation. One period of uniaxial cyclic deformation can be divided into two process according to the deformation mechanism, one is the tensile process in which the specimen is forcedly deformed, and the other is the recovery process in which the specimen recovers its deformation spontaneously. Furthermore, the viscoelasticity of polymers is a function of the imposed strain rate. During one period of sinusoidal imposed strain, the strain rate is different in the different stages or zones. So, it is reasonable to expect that the nonlinear dynamic viscoelasticity may exhibit different behavior at the different stages or steps during a period of cyclic deformation. In the present paper, the authors proposed an approach to analyze the nonlinear dynamic viscoelasticity at each divided cyclic strain zone, and the authors called this approach as "zone nonlinear dynamic viscoelastic analysis."

On the other hand, in order to extend the liquid crystalline polymer to the applications with large deformation or long-term bearing load, it is very important to know how the unique fatigue behavior of this new-type polymers is related with their very rigid polymer chains. 
However, no attempt has been made to reveal the relationship between the rigidity of polymer chains and the fatigue behavior for polymers. In this study, the relationships among the rigidity of polymer chains, the nonlinear dynamic viscoelasticity and the fatigue behavior for polymeric fibers were discussed on the basis of NVP analysis and zone NVP analysis under cyclic fatigue.

\section{EXPERIMENTAL}

\section{Specimens}

Two kinds of high-strength and high-modulus polymeric fibers with different polymer chain rigidity were used in this study. Vectran (a copolymer of $p$-hydroxy benzoate (HBA) and 2-hydroxy-6-naphthoic acid (HNA)) was the fiber with rigid polymer chains, and poly(vinyl alcohol) (PVA) was the fiber with flexible polymer chains. Figure 1 and Table I show their chemical structures and mechanical properties, respectively.

\section{Fatigue Tester with In-Situ Measurement for Nonlinear Dynamic Viscoelasticity}

Figure 2 shows the blockdiagram of the fatigue tester which enables the in-situ measurement of nonlinear dynamic viscoelasticity during fatigue process. The specimen of the single fiber was placed into a specimen chamber with temperature controller in a dry nitrogen purge. A sinusoidal strain was imposed to the specimen with a variable magnitude of strain amplitude at frequency of $11 \mathrm{~Hz}$. Both of the signals from the strain detector and the stress detector contained a static part $\left(\varepsilon_{\mathrm{s}}\right.$ or $\left.\sigma_{\mathrm{s}}\right)$ and a dynamic part $\left(\varepsilon_{\mathrm{d}}\right.$ or $\left.\sigma_{\mathrm{d}}\right)$ as shown in Figure 2. Due to the difficulties involved in the amplification of the signals with a DC component directly, the signals were separated into the static parts (DC component) and the dynamic parts (AC component), respectively by high-pass filters as shown in Figure 2. The amplified static signals were measured by digital multimeters and then, sent into a personal computer with GP-IB interface.

$$
\begin{aligned}
& +\underset{{ }_{\mathrm{OH}}}{+\mathrm{CH}_{2}-\mathrm{CH}}+\overbrace{\mathrm{n}} \\
& \text { PVA } \\
& +O-O-\stackrel{O}{C}_{(x / y=7 / 3)}^{O}+O-O-\stackrel{O}{C}_{y} \\
& \text { Vectran }
\end{aligned}
$$

Figure 1. Chemical Structures of PVA and Vectran fibers.

Table I. Mechanical properties of Vectran and PVA fibers ${ }^{a}$

\begin{tabular}{lcccc}
\hline & $d / \mu \mathrm{m}$ & $E / \mathrm{GPa}$ & $\sigma_{\mathrm{b}} / \mathrm{GPa}$ & $\varepsilon_{\mathrm{b}} / \%$ \\
\hline Vectran & 23 & 75 & 3.5 & 3.8 \\
PVA & 21 & 49 & 2.5 & 4.3 \\
\hline
\end{tabular}

${ }^{\mathrm{a}} d$, diameter of fiber; $E$, tensile modulus; $\sigma_{\mathrm{b}}$, breaking strength; $\varepsilon_{\mathrm{b}}$, breaking strain.
Also, the amplified dynamic signals were converted into digital data with a high sampling rate 2 channel $A / D$ converter and then, the converted digital data were sent into the computer and analyzed by fast Fourier transformation (FFT).

\section{Principle of Nonlinear Viscoelastic Parameter (NVP) Analysis}

Since the high-pass filters were used to separate the dynamic signals from the mixing signals, only the dynamic parts for strain and stress were analyzed below. When the uniaxial sinusoidal dynamic strain, $\varepsilon(t)$ with angular frequency, $\omega$ is imposed on the specimen (eq 1), the response stress, $\sigma(t)$ can be expanded by a Fourier series (eq 2).

$$
\begin{aligned}
\varepsilon(t)= & \varepsilon_{0} \sin (\omega t) \\
\sigma(t)= & \sigma_{1} \sin \left(\omega t+\delta_{1}\right)+\sigma_{2} \sin \left(2 \omega t+\delta_{2}\right)+\cdots \\
& +\sigma_{j} \sin \left(j \omega t+\delta_{j}\right)+\cdots
\end{aligned}
$$

where $\varepsilon_{0}$ is the imposed strain amplitude, and $\sigma_{1}$ and $\delta_{1}$ are the fundamental stress amplitude and the phase difference angle, respectively. $\sigma_{2}, \sigma_{3} \cdots$, and $\delta_{2}, \delta_{3} \cdots$ are the higher harmonic stress amplitudes and the higher harmonic phase difference angles, respectively. The nonlinear viscoelastic parameter, NVP, ${ }^{3-5}$ which enables the evaluation of the degree of nonlinear dynamic viscoelasticity, was defined as eq 3 .

$$
\mathrm{NVP}=\frac{\sigma_{2}+\sigma_{3}+\cdots+\sigma_{j}+\cdots+\sigma_{n}}{\sigma_{1}}
$$

where the number of higher harmonics, $n$ was taken into consideration up to 100 since the higher harmonics over 100 th are very small and can be ignored.

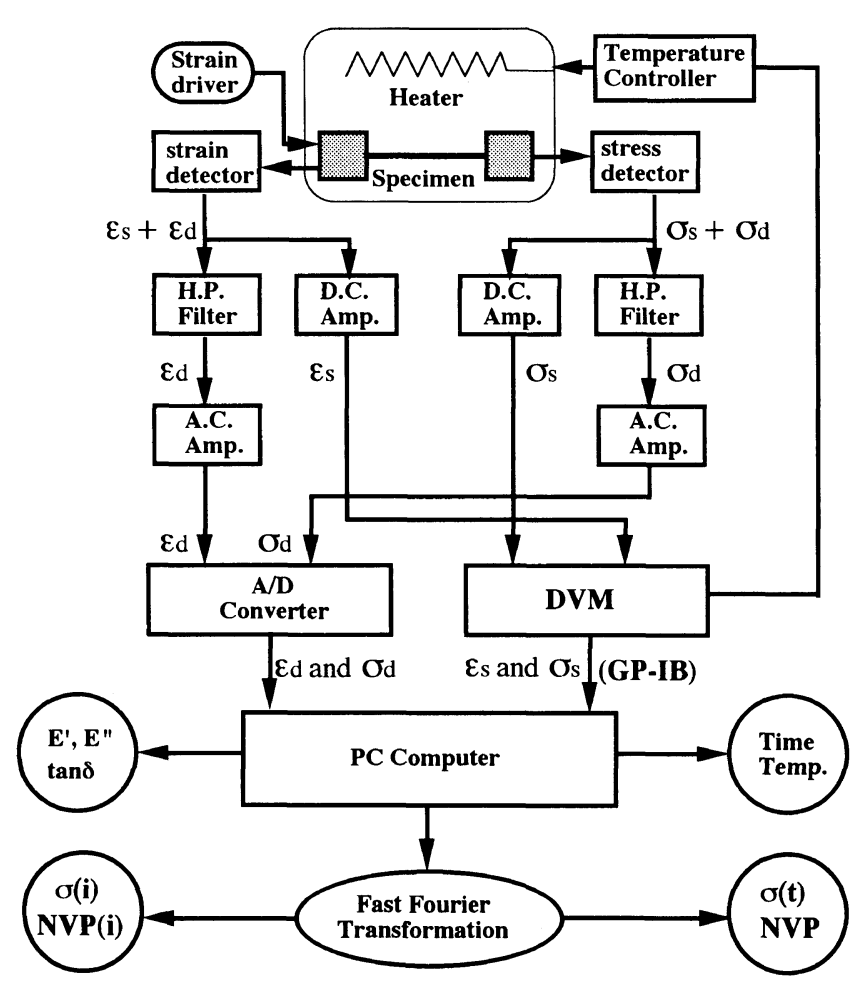

Figure 2. Blockdiagram of fatigue tester for investigation of nonlinear dynamic viscoelastic properties under cyclic fatigue test. 
Principle of Zone Nonlinear Dynamic Viscoelastic Analysis

Figure 3 shows the wave shapes of the imposed strain and the response stress for Vectran and PVA fibers at various strain amplitudes at $303 \mathrm{~K}$ after 100 seconds from the start of the fatigue test. As to the stress response, the magnitude of the deviation in the wave shape from sinusoidal imposed strain increased with an increase in the imposed strain amplitude. It indicated that the degree of nonlinear viscoelasticity increased with the magnitude of the imposed strain amplitude. Also, it could be found that the deviation of the wave shape of the stress response was different at the different stages or zones during one period of cyclic deformation. In order to give a detail description for the nonlinear response, the authors proposed the "zone nonlinear dynamic viscoelastic analysis." Figure 4 shows the principle of the zone nonlinear dynamic viscoelastic analysis. One period of cyclic deformation is divided into several zones. The variation of response stress with time in each zone can be represented by a Fourier expansion. If one period of cyclic deformation is divided into $2 m$ zones ( $m$ is an integer), that is, $m$ tensile zones and $m$ recovery zones, we can obtain $m$ equations for tensile process and other $m$ equations for recovery process as shown in eq 4 .

$$
\begin{aligned}
\sigma(i, t)= & \sigma_{1}(i) \sin \left[\omega t+\delta_{1}(i)\right]+\sigma_{2}(i) \sin \left[2 \omega t+\delta_{2}(i)\right]+\cdots \\
& +\sigma_{j}(i) \sin \left[j \omega t+\delta_{j}(i)\right]+\cdots
\end{aligned}
$$

where $i$ is the zone number such as T3, R4, etc., as shown in Figure 4. In this study, each period of cyclic deformation was divided into 12 zones $(i=\mathrm{T} 1, \cdots \mathrm{T} 6, \mathrm{R} 6, \cdots$ $\mathrm{R} 1)$, i.e., 6 tensile ( $\mathrm{T} 1 \cdots \mathrm{T} 6)$ and 6 recovery $(\mathrm{R} 1 \cdots \mathrm{R} 6)$ zones. Then, the zone nonlinear viscoelastic parameter, $\operatorname{NVP}(i)$, which represents the degree of nonlinear dynamic viscoelasticity in the $i$ th zone, is defined as eq 5 .

$$
\operatorname{NVP}(i)=\frac{\sigma_{2}(i)+\sigma_{3}(i)+\cdots+\sigma_{j}(i)+\cdots+\sigma_{n}(i)}{\sigma_{1}(i)}
$$

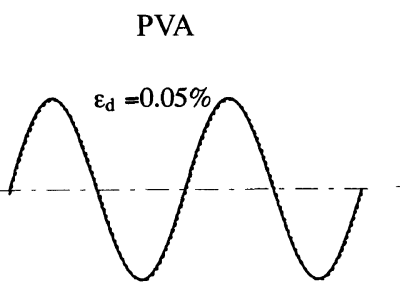

\section{Vectran}
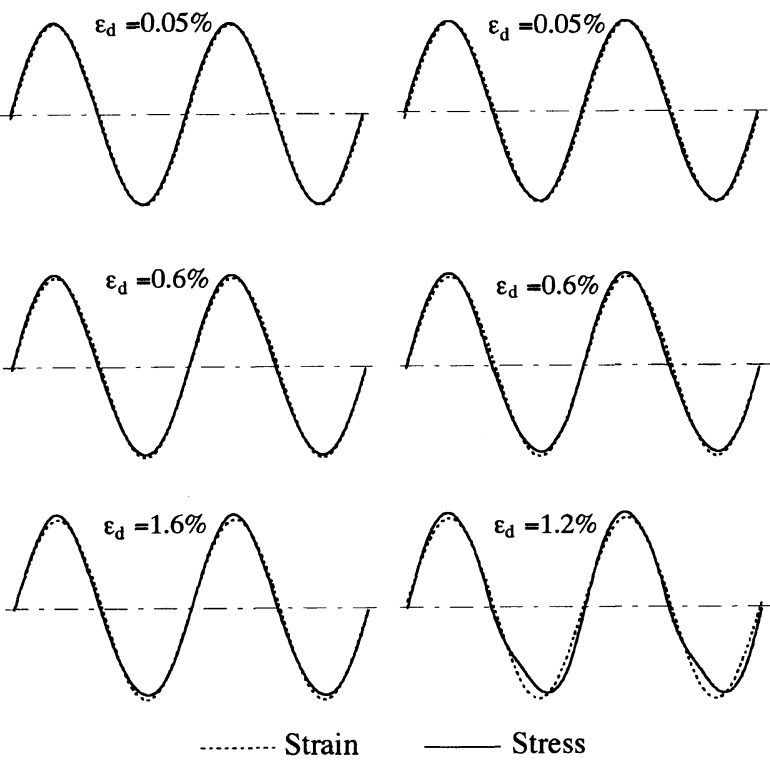

Figure 3. Wave shapes of imposed strain and response stress for Vectran and PVA fibers at various strain amplitudes at $303 \mathrm{~K}$ after 100 seconds from the start of the fatigue test. where the number of higher harmonics, $n$ is taken into consideration up to 100 as mentioned in eq 3 .

\section{Wide Angle X-Ray Diffraction}

Wide angle X-ray diffraction (WAXD) experiments were carried out for the polymeric fibers using a X-ray generator (M18XHF-SRA, Mac Science Co., Ltd.) with graphite filtered $\mathrm{Cu}-K_{\alpha}$ radiation $(\lambda=0.15405 \mathrm{~nm})$ at room temperature. X-Ray diffraction patterns were recorded with an imaging plate (DIP2000, Mac Science Co., Ltd.). A bundle of parallel fibers was fixed on a metal frame that had a freely sliding side to extend the fibers up to various degree of strains.

\section{Scanning Electron Microscopic Observation}

Scanning electron microphotographs (SEM) of polymeric fibers weretaken by using a Hitachi S-2120 scanning electron microscope. The polymeric fibers that were unsubjected and subjected to fatigue test were observed by SEM.

\section{RESULTS AND DISCUSSION}

\section{Relationship between Nonlinear Dynamic Viscoelasticity and Fatigue Behavior}

Figure 5 shows the relationships between the average value of NVP during fatigue test and fatigue lifetime for Vectran and PVA fibers under various imposed dynamic strain amplitudes at $303 \mathrm{~K}$. The fatigue lifetime of PVA fiber which exhibited smaller NVP value was much longer than that of Vectran fiber which exhibited larger NVP value under the same imposed strain condition.

From the results shown in Figure 5, it seemed reasonable to conclude that the fatigue lifetime became shorter with an increase in the magnitude of NVP. This con-
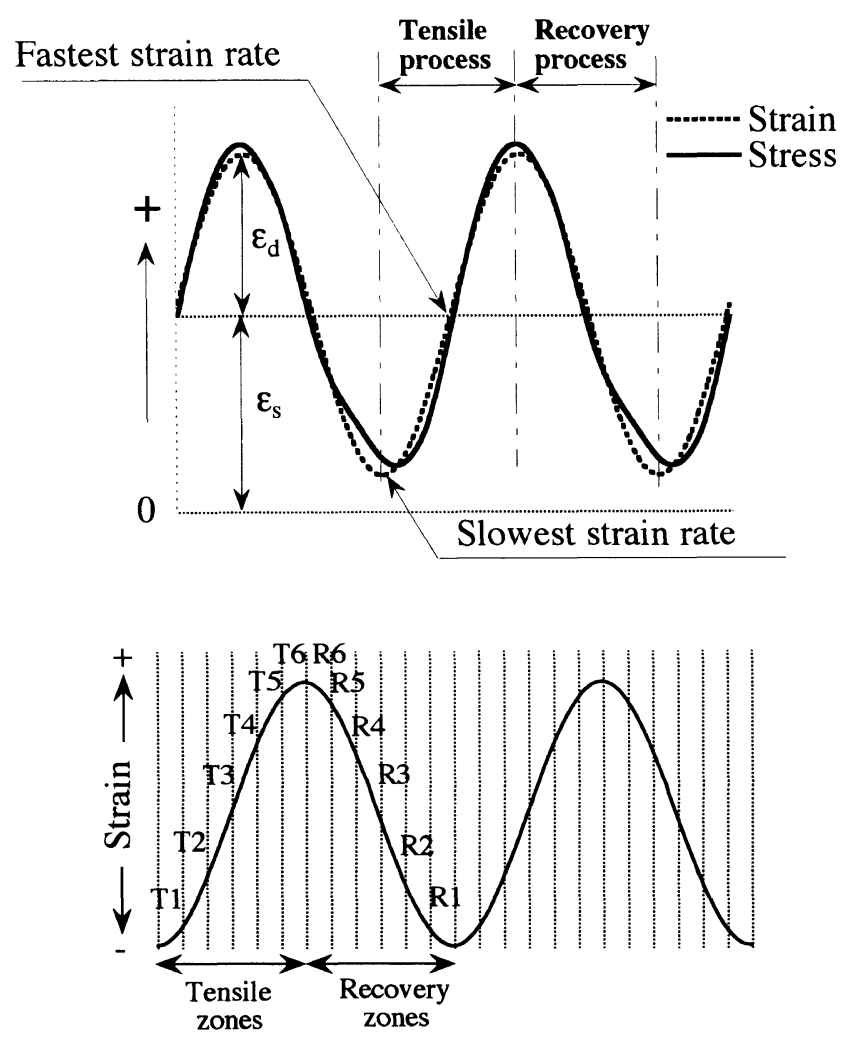

Figure 4. Principle of zone nonlinear dynamic viscoelastic analysis. 


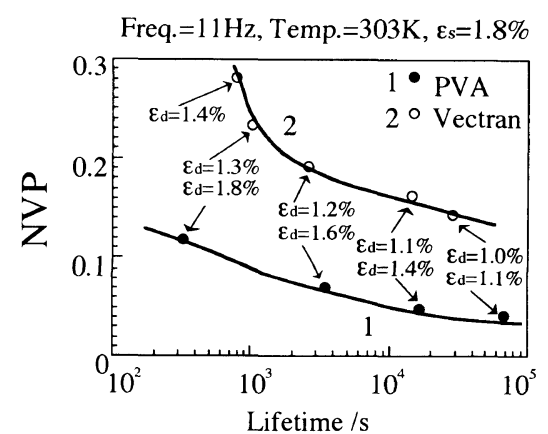

Figure 5. Relationships between the average value of NVP during fatigue process and fatigue lifetime for Vectran and PVA fibers under various imposed strain amplitudes at $303 \mathrm{~K}$.

clusion was well in agreement with that made by our previous papers ${ }^{3,4}$ in the case of polyethylene. It was considered that since the irreversible structural changes would occur more frequently when the specimen exhibited greater nonlinear viscoelasticity, the fatigue deterioration induced by the irreversible structural changes would grow faster, and finally resulted in a shorter fatigue lifetime.

\section{Strain Amplitude Dependence of Nonlinear Viscoelastic Parameter (NVP)}

Figure 6 shows the variations of NVP with the imposed dynamic strain amplitudes for PVA and Vectran fibers at $303 \mathrm{~K}$ after 100 seconds from the start of fatigue test. NVP value increased with the magnitude of the imposed strain amplitude for both PVA and Vectran fibers. Vectran fiber with rigid polymer chains exhibited much greater nonlinear viscoelasticity than PVA fiber with flexible polymer chains. Also, the NVP value of Vectran fiber increased very steeply with the imposed strain amplitude in comparison with that of PVA fiber. These results suggested that the rigidity of polymer chains exerted a great effect on the nonlinear dynamic viscoelasticity for polymers.

There was still lack of data to give a satisfactory interpretation about why the remarkable nonlinear viscoelasticity was exhibited in the case of the rigid chain polymers. The authors suggested that the aggregation structure of polymers may play an important role here. In general, crystalline polymers such as polymeric fibers have a relatively hard crystal region comparing with a relatively soft amorphous region in aggregation structure. This means that the imposed deformation will be predominantly absorbed by the amorphous region rather than by the crystalline region. ${ }^{10,11}$ In the case of polymer with rigid polymer chains (Vectran), since its amorphous region was almost as hard as its crystalline region, ${ }^{10}$ the imposed strain could not be absorbed by the soft amorphous region as easy as the case of the polymer with flexible polymer chains. So, the irreversible structural change such as the micro-slippage among polymer chains and/or the macro-slippage among microfibrils would occur more easily or more frequently, and such irreversible structural change would lead to the remarkable nonlinear dynamic viscoelastic behavior to be exhibited for the rigid chain polymers.

In order to estimate the degree of deformation occurred in the crystalline region, the wide angle $\mathrm{X}$-ray

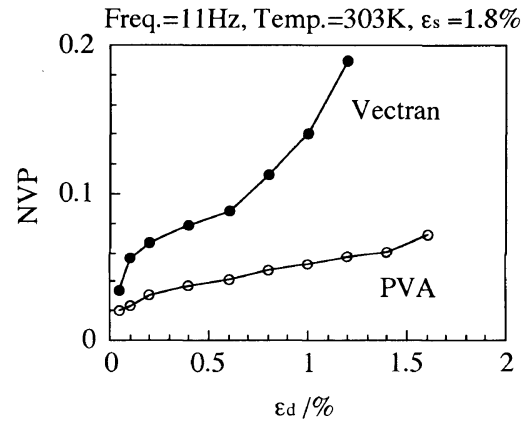

Figure 6. Variations of NVP with imposed dynamic strain amplitude for Vectran and PVA fibers at $303 \mathrm{~K}$ after 100 seconds from the start of fatigue test.

Table II. The actual strain in crystalline region $\left(\varepsilon_{\mathrm{c}}\right)$ and the strain of the specimen $(\varepsilon)$ for Vectran and PVA fibers at room temperature

\begin{tabular}{lllll} 
& $\varepsilon / \%$ & 0 & 1.5 & 3 \\
\hline Vectran & $\varepsilon_{\mathrm{c}} / \%$ & 0 & 0.56 & 1.40 \\
\hline PVA & $\varepsilon_{\mathrm{c}} / \%$ & 0 & 0.17 & 0.51 \\
\hline
\end{tabular}

diffraction experiments were carried out for Vectran and PVA fibers under tension condition. The degree of deformation occurred in the crystalline region, $\varepsilon_{\mathrm{c}}$ was evaluated by eq $6 .^{12}$

$$
\varepsilon_{\mathrm{c}}=\frac{\Delta d_{h k l}}{d_{h k l}}
$$

where, $\varepsilon_{\mathrm{c}}$ is the magnitudes of crystal strain. Also, $\Delta d_{h k l}$ is the variation of the plan distance of $(h k l), d_{h k l}$ under the strain of the specimen, $\varepsilon$. Furthermore, in order to calculate $\varepsilon_{\mathrm{c}}$ along the direction parallel to the fiber axis, the $(002)$ and $(001)$ reflections were used for Vectran and PVA fibers, respectively. Table II shows the variations of $\varepsilon_{\mathrm{c}}$ under the various imposed static strain, $\varepsilon$. The small $\varepsilon_{\mathrm{c}}$ in the case of PVA indicated that the deformation in the amorphous region was much larger than that in the crystalline region. By contrast, the larger $\varepsilon_{\mathrm{c}}$ in the case of Vectran fiber indicated that the deformation could not be absorbed so easily by the amorphous regions as the case of PVA fiber. These results were in good agreement with our consideration mentioned above.

\section{Zone Nonlinear Dynamic Viscoelastic Analysis}

Figure 7 shows the zone nonlinear dynamic viscoelastic behavior for Vectran and PVA fibers under various imposed dynamic strain amplitudes at $303 \mathrm{~K}$ after 100 seconds from the start of fatigue test. The abscissa corresponds to the zone number shown in Figure 4 and the ordinate is the magnitude of $\operatorname{NVP}(i)$. The $\operatorname{NVP}(i)$ values during recovery zones were greater than those during tensile zones for both Vectran and PVA fibers. This result suggested that the nonlinear dynamic viscoelasticity predominantly appeared during recovery zones rather than tensile zones. Also, the result shown in Figure 7 suggested that the rigidity of polymer chains strongly affected their zone nonlinear viscoelastic behavior. Vectran fiber exhibited greater nonlinear viscoelasticity than PVA fibers in each deformation zone, and the nonlinear viscoelastic behavior during the recovery zones was 


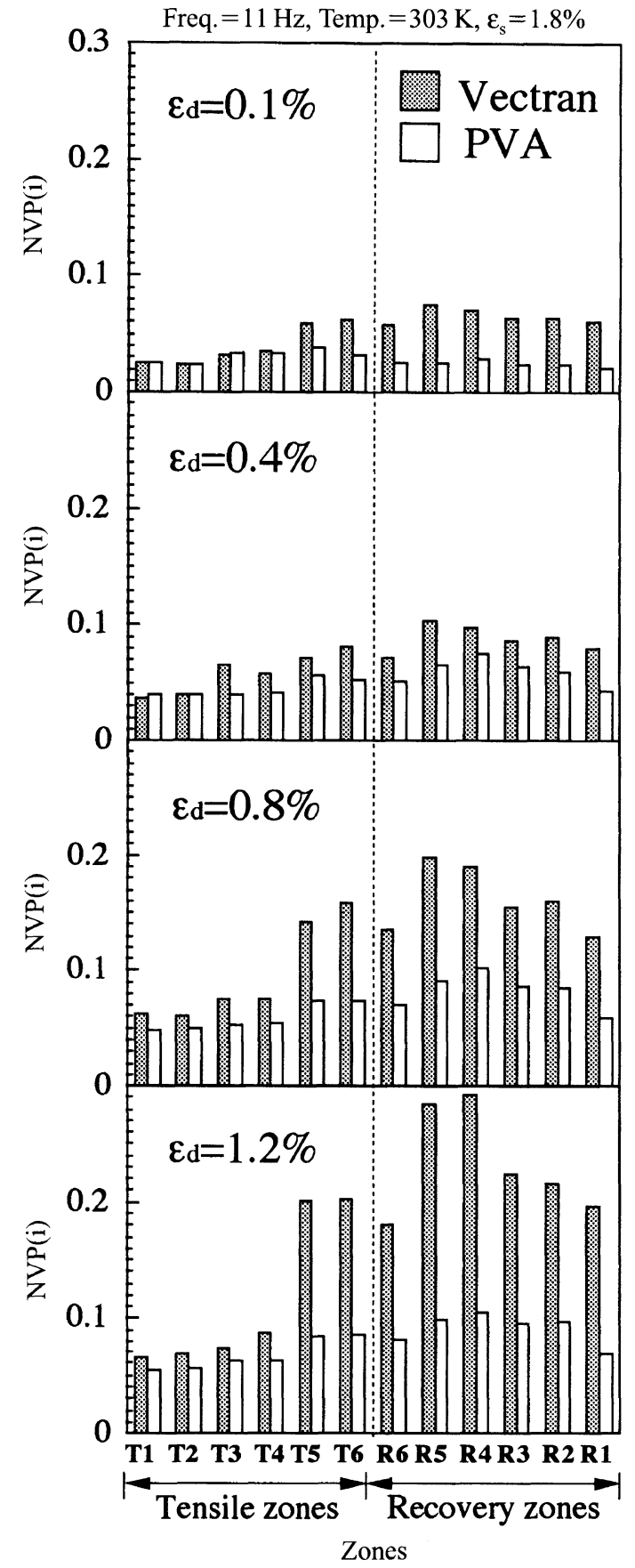

Figure 7. Comparison of zone nonlinear viscoelastic parameter, $\operatorname{NVP}(i)$ for Vectran and PVA fibers at 303K after 100 seconds from the start of fatigue test.

especially remarkable in the case of Vectran fiber. Also, it should be noticed that in the zone with higher strain rate, the $\operatorname{NVP}(i)$ value was often larger than that in the zone with lower strain rate (strain rate: $\mathrm{T} 1=\mathrm{T} 6<\mathrm{T} 2=$ $\mathrm{T} 5<\mathrm{T} 3=\mathrm{T} 4 ; \mathrm{R} 1=\mathrm{R} 6<\mathrm{R} 2=\mathrm{R} 5<\mathrm{R} 3=\mathrm{R} 4)$.

It was interested that the rigid chain polymer exhibited very remarkable nonlinear viscoelasticity during the recovery process. We supposed that this might be related to the kink-band formation. It has been well known that the kink-band formation usually occurs under compressive deformation for the rigid chain polymers, and this results in the poor compressive strength in comparison with their excellent tensile strength. ${ }^{13}$ It seems reasonable to consider that the recovery process during cyclic

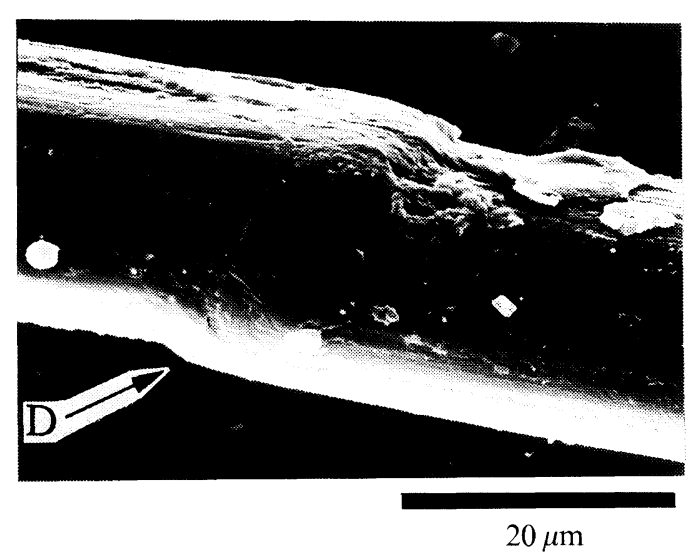

(a) Vectran fiber

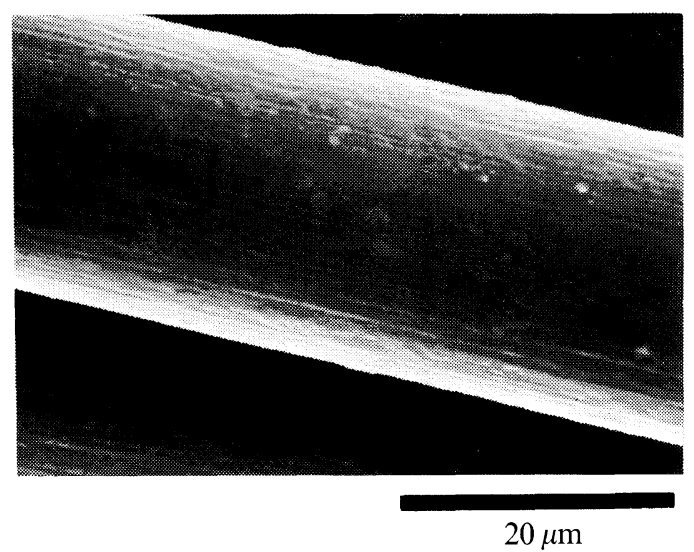

(b) PVA fiber

Figure 8. Scanning electron micrographs for Vectran and PVA fibers after 100 seconds from the start of fatigue test under $\varepsilon_{\mathrm{d}}=1.2 \%, \varepsilon_{\mathrm{s}}=$ $1.8 \%$ at $303 \mathrm{~K}$. The portion " $\mathrm{D}$ " indicates a kink band.

deformation is similar to the compressive deformation. So the micro-kinking among polymer chains and/or the macro-kinking among microfibrils might occur frequently during the recovery process. Furthermore, the remarkable nonlinear viscoelastic behavior during the recovery zones might be induced by such kink-band formation in the case of the rigid chain polymer.

Figure 8 is a scanning electron micrographs for Vectran and PVA fibers after 100 seconds from the start of fatigue test. The kink-band formation in the case of Vectran fiber was observed, however, no evident kink-band formation was observed in the case of PVA fiber. So the kink-band formation during the recovery process might be one of the reasons why the rigid chain polymer exhibited very remarkable nonlinear viscoelasticity during the recovery zones.

\section{Variation of Zone Nonlinear Viscoelastic Behavior with Time during Fatigue Process}

Figures 9 show the variations of $\operatorname{NVP}(i)$ value with time during fatigue process for Vectran (Figure 9(a)) and PVA(Figure 9(b)) fibers at $303 \mathrm{~K}$. During the fatigue process, the NVP $(i)$ value did not show significant variation until the onset of fatigue failure in both cases of Vectran and PVA fibers. At the onset of fatigue failure, an abrupt increase in the $\operatorname{NVP}(i)$ value was observed for 


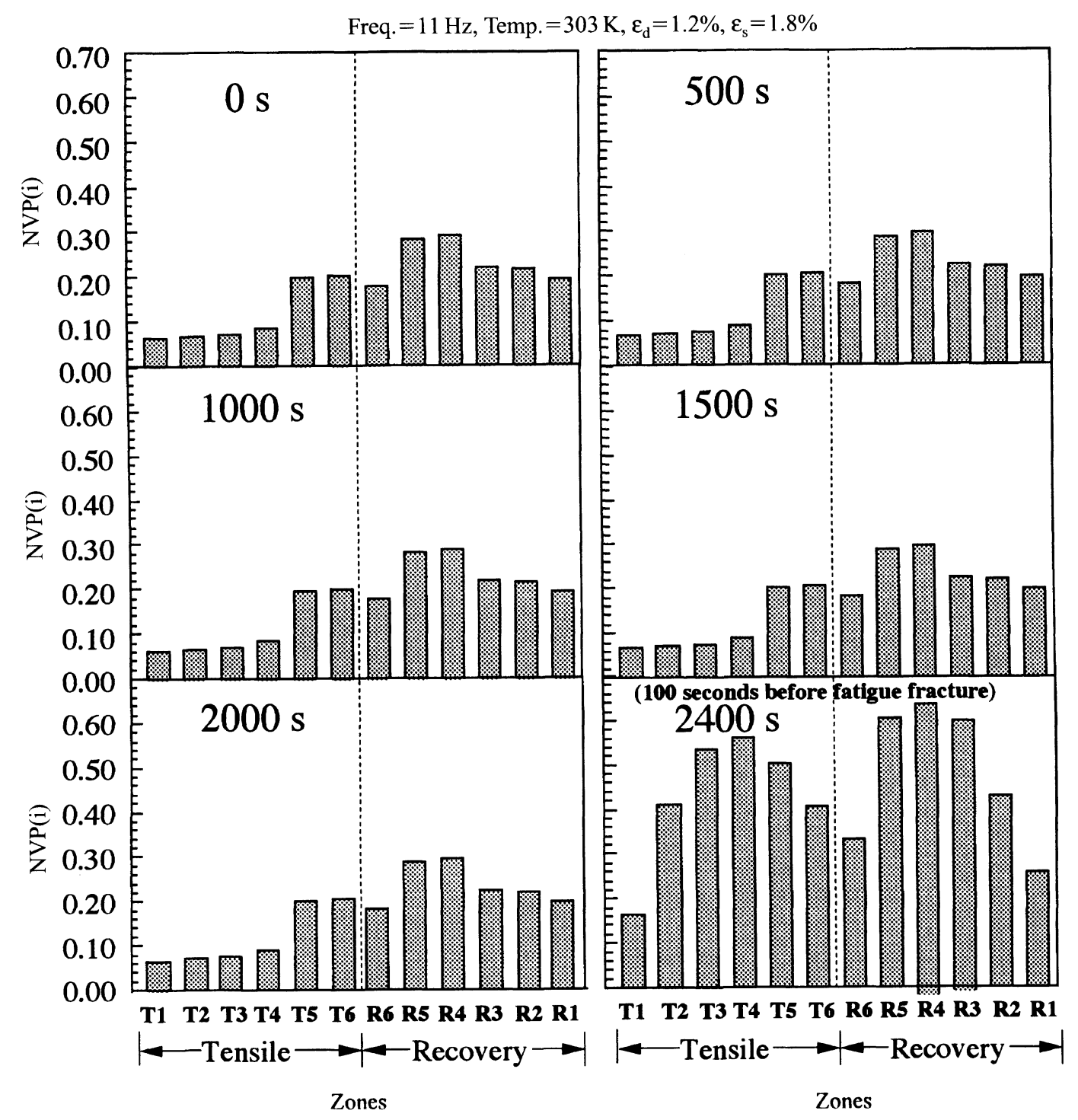

(a) Vectran

Figure 9(a)

both fibers. It was important to note that the fashion of the increase in NVP $(i)$ caused by increasing the imposed strain amplitude was much different from that caused by fatigue (see Figures 7 and 9). In the former case, the increase of $\mathrm{NVP}(i)$ was predominant in recovery zones, but in the latter case, the increase of $\operatorname{NVP}(i)$ was observed in both tensile and recovery zones, especially in the zone with higher strain rate. Such increase in NVP $(i)$ before fatigue failure was considered to be related to the remarkable irreversible structural changes caused by cyclic deformation.

\section{CONCLUSION}

The fatigue behavior was investigated on the basis of NVP and zone NVP analyses for the polymeric fibers with different main-chain rigidity. The polymeric fiber with rigid polymer chains showed greater nonlinear dynamic viscoelasticity and poorer fatigue strength than that with flexible polymer chains. The zone nonlinear dynamic viscoelastic analysis revealed that the nonlinear viscoelastic behavior was predominantly induced during the recovery process rather than the tensile process for oriented polymers such as polymeric fibers. It was considered that the strong nonlinear viscoelasticity and the poor fatigue strength in the case of the rigid chain polymers might be attributed to the ease of the slippage and the kink band formation among the rigid polymer chains.

\section{REFERENCES}

1. J. A. Sauer and M. Hara, Adv. Polym. Sci., 91/92, 71 (1990).

2. A. Takahara, T. Magome, and T. Kajiyama, J. Polym. Sci., Polym. 

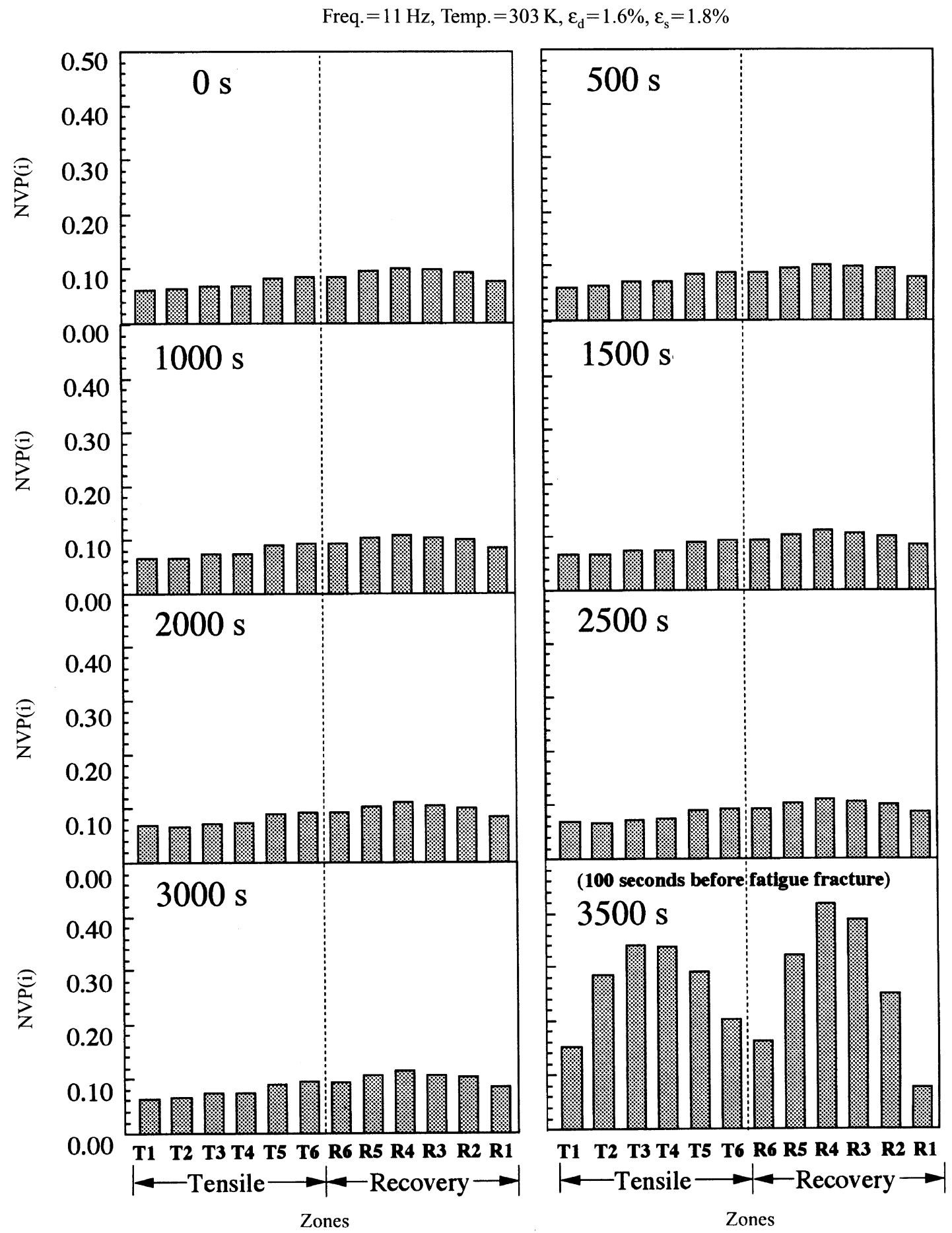

(b) PVA

Figure $9(\mathrm{~b})$

Figure 9. Variations of $\mathrm{NVP}(i)$ with time during fatigue process for (a) Vectran and (b) PVA fibers at $303 \mathrm{~K}$.

Phys. Ed., 32, 839 (1994).

3. N-J. Jo, A. Takahara, and T. Kajiyama, Polym. J., 25, 721 (1993)

4. N-J. Jo, A. Takahara, and T. Kajiyama, Polym. J., 26, 1024(1994).

5. T. Liang, K. Tokunaga, A. Yamashita, A. Tahahara, and T. Kajiyama, Polym Bull., 36, 477 (1996).

6. D. C. Prevorsek, Y. D. Kwon, and R. K. Sharma, J. Macromol. Sci. Phys., B13, 571 (1977)

7. D. C. Prevorsek, Y. D. Kwon, and R. K. Sharma, J. Appl. Polym.
Sci., 25, 2063 (1980).

8. W. C. MacSporran and R. P. Spiers, Rheol. Acta, 23, 90 (1984).

9. A. Tanaka, M. Fukuda, H. Nagai, M. Shinohara, and S. Onogi, J. Polym. Sci., Polym. Phys. Ed., 27, 2283 (1989).

10. K. Yabuki, H. Ito, and T. Ota, Sen-i Gakkaishi, 32, T58 (1976).

11. C. Vattas and C. Galiotis, Polymer, 35, 2335 (1994).

12. W. J. Dulmage and L. E. Contois, J. Polym. Sci., 8, 275 (1958).

13. F. J. Wortmann and K. V. Schulz, Polymer, 35, 2108 (1994). 